\title{
Capacidad de carga estática de una viga armada tipo I con soldadura intermitente en la unión alma-patín
}

\author{
Carlos Naranjo-Guatemala, Xavier Nicolalde-Espinosa, María Alcívar-Vaca \\ Departamento de Ciencias de la Energía y Mecánica, Universidad de las Fuerzas Armadas ESPE \\ crnaranjo@espe.edu.ec,mjalcivar1@espe.edu.ec, fxnicolalde@espe.edu.ec
}

\begin{abstract}
Resumen-Para optimizar el uso tanto de materiales como de mano de obra empleados en la fabricación de vigas secundarias, se estudia si la variación de porcentaje de soldadura en la unión alma-patín altera la capacidad de carga de la viga. Se diseñaron tres vigas de igual geometría, pero con la variación de que la conexión viga patín tenían un porcentaje de soldadura del $70 \%, 57 \%$ y $37 \%$. Las vigas cumplieron los criterios establecidos en los códigos NEC-SE-AC y AWS D1.1. Las vigas diseñadas fueron simuladas en un software CAE, las vigas construidas se sometieron a un ensayo a flexión bajo las mismas condiciones de carga y apoyos. El valor del esfuerzo normal, esfuerzo cortante y las deflexiones experimentales se compararon con los obtenidos en el modelo computacional y los que establece la formulación teórica. Adicional a esto se efectúo un análisis del costo comparativo de la fabricación de vigas con soldadura intermitente y continua, tomando como parámetros de variación los consumibles de soldadura y la mano de obra.
\end{abstract}

Palabras Claves-Soldadura intermitente, viga armada, flexión, esfuerzos flectores, deflexión

\begin{abstract}
To optimize the use of both materials and workforce used in manufacturing of secondary beams, it is studied if the variation of percentage of welding in the webflange union does not alter the load capacity of the element. Beams of the same geometry but with different percentage of seams weld were designed according the standards NEC-SEAC and AWS D1.1, These beams were simulated in CAD / CAE software. The simulated and constructed beams have a welding percentage of $70 \%, 57 \%$ and $37 \%$. The constructed beams were submitted to a bending test under the same conditions of load and supports, the experimental results were compared with those obtained in the computational and theoretical model, the analysis of stresses and deformation of each beam was performed. In addition to this, a comparative economic analysis of the manufacture of beams with intermittent and continuous welding is presented, taking as parameters of variation the welding consumables and workforce.
\end{abstract}

Keywords - Intermittent welding, reinforced beam, bending, bending efforts, deflection

\section{INTRODUCCIÓN}

Uno de los elementos a optimizar en la industria de la construcción es el uso de materiales y mano de obra, a fin de reducir el costo y tiempo de ejecución de un proyecto, así como la afectación al medio ambiente [1].

La distribución teórica de los esfuerzos cortantes en una viga secundaria indica que en la unión del alma con los patines los valores de estos son reducidos, por lo cual se puede inferir que un cordón intermitente puede cumplir satisfactoriamente con la solicitación de cargas sin afectar la resistencia de la viga [6].
Reza M, (2006) estudio el comportamiento de cordones de filete intermitentes en una unión placa-refuerzo sometido a cargas perpendiculares al cordón. Se hace referencia a la reducción de costos de mano de obra, materiales en la fabricación, así como a la reducción de tensiones residuales y distorsiones al utilizar soldadura intermitente en lugar de continua como método de fabricación; la reducción de tensiones se da debido a que la longitud de la soldadura y la energía de entrada de calor en la soldadura intermitente es menor que en el caso de la soldadura continua. [8]

Sin embargo, el uso de soldadura intermitente puede resultar en una reducción significativa en la capacidad de carga dinámicas si se compara con una soldadura continua [9], por lo que este estudio se limita al análisis de elementos que no se encuentren sometidos a fatiga.

El presente trabajo estudia la unión alma-patín soldada con cordones de filete de varios valores de intermitencia, considerando los valores límites establecidos para soldadura intermitentes en los códigos NEC-SE-AC y AWS D1.1.

El estudio se realizó en tres vigas en los que se midió: esfuerzo normal, esfuerzo cortante y deflexión. Estos resultados fueron comparados con los obtenidos en modelos computacionales y teóricos para verificar su validez.

\section{DiseÑo DE LA VigA}

\section{A. Diseño Geométrico de la Viga}

Requisitos de diseño:

- Viga de sección tipo I compacta.

- El modo de fallo debe ser por flexión.

- La carga crítica será puntual y estará aplicada en la mitad de la viga con un valor de 6 toneladas.

- La soldadura debe ser de filete con una junta en T

- La viga estará simplemente apoyada.

- El material de la viga será acero estructural ASTM A36.

- Una longitud máxima de 3 metros.

Con base al código NEC-SE-AC [7] y la especificación ANSI/AISC 360-10 [2], se obtuvo las medidas de la sección de viga como se muestra en la Fig. 1. 


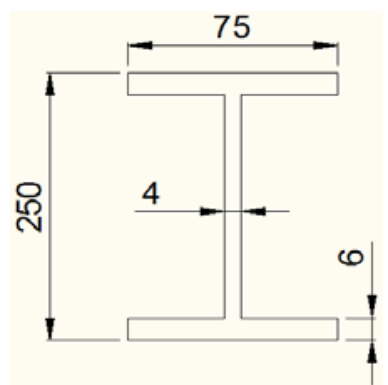

Fig. 1. Sección de la viga.

\section{B. Especificación del Procedimiento de Soldadura}

Se realizó en base a un procedimiento precalificado conforme a lo establecido en el código AWS D1.1 [3] y los parámetros principales del proceso se muestran en la Tabla I. El cordón de soldadura se especificó en un filete de $5 \mathrm{~mm}$ de pierna.

TABLA I

PARÁMETROS PARA EL PROCEDIMIENTO DE SOLDADURA

\begin{tabular}{lc}
\hline \hline \multicolumn{1}{c}{ Proceso } & GMAW \\
\hline Material de aporte: clase/diámetro $(\mathrm{mm})$ & ER70S-6 / 0.8 \\
Gas de protección & Argón/CO2 \\
Corriente (A) & $130-160$ \\
Voltaje (V) & $20-22$ \\
Velocidad de avance $(\mathrm{cm} / \mathrm{min})$ & $42-46$ \\
Velocidad de alimentación $(\mathrm{cm} / \mathrm{min})$ & $380-420$ \\
\hline \hline
\end{tabular}

\section{Diseño de la Conexión Soldada - Intermitencia de Soldadura}

El párrafo 2.12.2.2 del código AWS D1.1, establece que el espacio máximo entre cordones de soldadura intermitente es de $E s=100 \mathrm{~mm}$ [3]. De todas las posibles combinaciones de cordón e intermitencia, se consideró vigas con cordones de $37 \%, 57 \%$ y $70 \%$ de longitud soldada, cuyos valores se detallan en Tabla II.

TABLA II

DATOS DE LA INTERMITENCIA DE SOLDADURA EN CADA CASO DE ESTUDIO

\begin{tabular}{ccccc}
\hline \hline $\begin{array}{c}\text { Espacio } \\
\text { entre } \\
\text { soldaduras } \\
\text { Es [mm] }\end{array}$ & $\begin{array}{c}\text { Número } \\
\text { espacios } \\
\text { \#e }\end{array}$ & $\begin{array}{c}\text { Número } \\
\text { de } \\
\text { cordones } \\
\text { \#c }\end{array}$ & $\begin{array}{c}\text { Longitud } \\
\text { del } \\
\text { cordón Lc } \\
\text { [mm] }\end{array}$ & $\begin{array}{c}\text { Porcentaje } \\
\text { de } \\
\text { soldadura } \\
{[\%]}\end{array}$ \\
\hline 100 & 9 & 10 & 210 & 70 \\
100 & 13 & 14 & 121 & 57 \\
100 & 19 & 20 & 55 & 37 \\
\hline \hline
\end{tabular}

\section{Modelos teóricos de cálculo de vigas.}

La teoría de vigas establece los siguientes modelos matemáticos para el cálculo de vigas simplemente apoyadas con carga en el centro de la viga [4], [5].

La deformación máxima:

$$
\delta_{\max }=\frac{P \cdot L_{r}^{3}}{48 \cdot E \cdot I_{x}}
$$

donde $P$ es la fuerza aplicada, $E$ es el módulo de elasticidad del material, $L_{r}$ es la longitud entre apoyos e $I_{x}$ es la inercia respecto al eje mayor

El esfuerzo cortante:

$$
\tau=\frac{P \cdot Q}{I_{x} \cdot b}
$$

donde $Q$ es el momento estático de primer orden y $b$ es el ancho del alma

El esfuerzo normal:

$$
\sigma_{n}=\frac{P \cdot L_{r}}{4 \cdot Z_{x}}
$$

Donde $Z_{x}$ es el módulo plástico de la sección

\section{SIMULACIÓN}

Con las condiciones del diseño se simularon en software CAE el comportamiento de las vigas con diferente porcentaje de soldadura. Los valores de deformación máxima, esfuerzo normal y esfuerzo cortante se registraron para su análisis.

En la Fig. 2 se observa que no todos los cordones de soldadura están sometidos a los mismos esfuerzos y deformaciones, presentado los mayores requerimientos los cordones ubicados en la mitad de la viga.

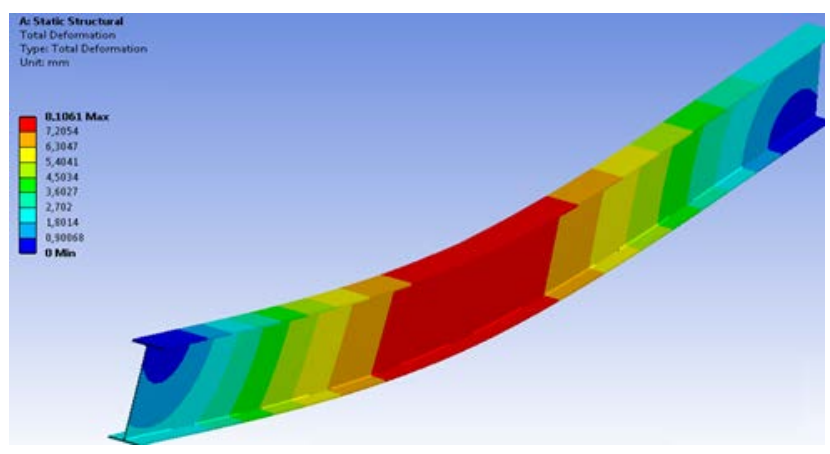

Fig. 2. Deformación total.

\section{CONSTRUCCIÓN DE LOS MODELOS REALES}

\section{A. Construcción de las Vigas}

Los 3 modelos para experimentar, con 70, 57 y 37 por ciento de cordones de soldadura, se construyeron conforme los diseños a cargo de un soldador experimentado para garantizar el tamaño y longitud de los cordones de soldadura.

\section{B. Arriostramiento o Apoyos Laterales}

Para garantizar que el modo de fallo del elemento sea por flexión y no por pandeo lateral torsional, se diseñó apoyos laterales [6], en base a lo establecido en la norma ANSI/AISC 360-10.

\section{Instrumentación de la Viga.}

Para medir la deformación se utilizó galgas extensiométricas de tipo lineal con una resistencia de 120 Ohmios y una tolerancia de $\pm 0.35 \%$, ubicadas en la mitad de la viga a unos 5 milímetros por encima del cordón inferior.

La deformación se midió con un comparador de reloj analógico de resolución $0.01 \mathrm{~mm}$, el cual se ubicó en el punto medio de la viga.

Los desplazamientos laterales se midieron con calibrador pie de rey de $150 \mathrm{~mm}$ con resolución de $0.02 \mathrm{~mm}$, en la Fig. 3 se muestra la ubicación de los puntos de medición. 


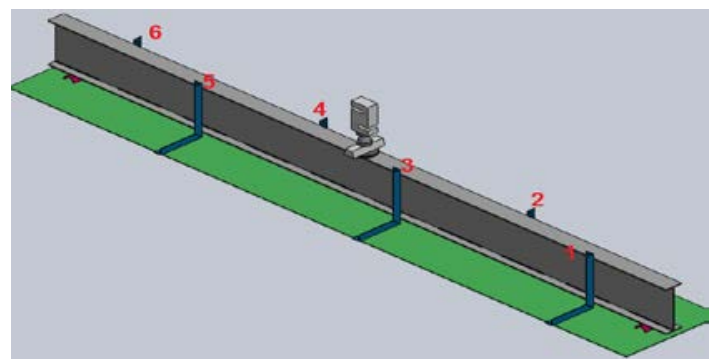

Fig. 3. Numeración de los apoyos.

\section{Ensayo de Flexión}

Las vigas se ensayaron conforme se visualiza en la Fig. 4. Durante el ensayo se midió la deformación unitaria, deformación máxima y desplazamiento lateral de la viga con respecto a los apoyos.

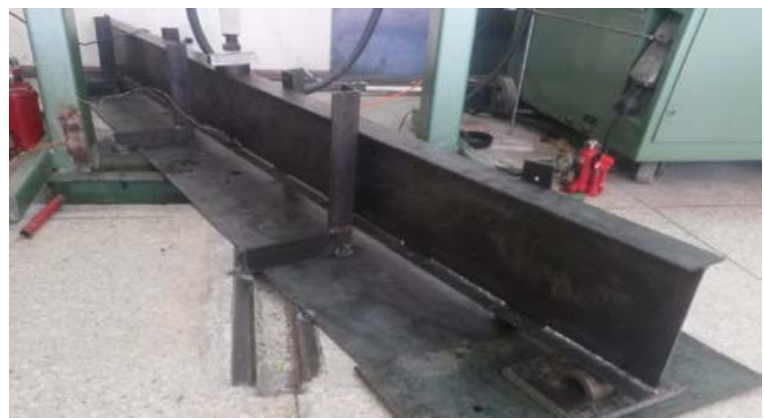

Fig. 4. Ensayo a flexión.

\section{Resultados}

\section{A. Modelo Teórico}

Los resultados teóricos para cualquier viga de la misma geometría y material son iguales independientemente de la intermitencia de los cordones de soldadura y son: $\sigma_{n}=248.11 \mathrm{MPa}, \tau=22.55 \mathrm{MPa}$ y $\delta=7.28 \mathrm{~mm}$.

\section{B. Simulación con $C A E$}

En la Tabla III se muestra los resultados obtenidos con el modelo de simulación con el software CAE, de las tres vigas.

TABLA III

RESULTADOS DE LAS SIMULACIONES PARA LAS VIGAS CONSTRUIDAS

\begin{tabular}{ccccc}
\hline \hline & $\begin{array}{c}\text { Porcentaje } \\
\text { de } \\
\text { soldadura } \\
(\%)\end{array}$ & $\begin{array}{c}\text { Deflexión } \\
\text { Máxima } \\
(\boldsymbol{\delta})[\mathbf{m m}]\end{array}$ & $\begin{array}{c}\text { Esfuerzo } \\
\text { Normal } \\
\text { Máximo } \\
\left(\boldsymbol{\sigma}_{\boldsymbol{n}}\right)[\mathbf{M P a}]\end{array}$ & $\begin{array}{c}\text { Esfuerzo } \\
\text { Cortante } \\
\text { Alma-Patín } \\
(\boldsymbol{\tau})[\mathbf{M P a}]\end{array}$ \\
\hline viga 1 & 37 & 8,18 & 265,27 & 24,35 \\
viga 2 & 57 & 8,14 & 263,81 & 24,02 \\
viga 3 & 70 & 8,11 & 263,64 & 24,78 \\
\hline \hline
\end{tabular}

\section{Modelo Experimental}

La Tabla IV presenta la deflexión y la deformación unitaria medida, valores con los que se calcula los esfuerzos normales y cortantes que fueron calculados a partir de la deformación unitaria de cada viga cuando eran sometidas a la carga máxima de $6000 \mathrm{~kg}$.
TABLA IV

RESUlTADOS OBTENIDOS A PARTIR DE LA EXPERIMENTACIÓN

\begin{tabular}{|c|c|c|c|c|c|}
\hline & $\begin{array}{c}\text { Porcen. } \\
\text { de } \\
\text { soldad. } \\
\text { (\%) }\end{array}$ & $\begin{array}{l}\text { Deflex. } \\
\text { Máxima } \\
(\delta)[\mathrm{mm}]\end{array}$ & $\begin{array}{c}\text { Deform. } \\
\text { Unitaria } \\
\varepsilon \\
{\left[\mathrm{x10}^{-6}\right]}\end{array}$ & $\begin{array}{c}\text { Esfuerzo } \\
\text { Normal } \\
\text { Máximo } \\
\left(\sigma_{n}\right) \\
{[\mathrm{MPa}]}\end{array}$ & $\begin{array}{c}\text { Esfuerzo } \\
\text { Cortante } \\
\text { Alma- } \\
\text { Patín } \\
(\boldsymbol{\tau}) \\
\text { [MPa] }\end{array}$ \\
\hline viga 1 & 37 & 8,39 & 1223 & 256,83 & 23,42 \\
\hline viga 2 & 57 & 8,29 & 1193 & 250,53 & 22,85 \\
\hline viga 3 & 70 & 7,81 & 1195 & 250,95 & 22,89 \\
\hline
\end{tabular}

\section{ANÁlisis DE RESUltAdOS}

A. Comparación de Resultados entre Modelo Teórico, Experimental y Computacional

La Tabla VI muestra las variaciones de los resultados entre los modelos utilizados.

Se observa en la Tabla $\mathrm{V}$ que la variación de los resultados de la simulación CAE respecto a los resultados obtenidos experimentalmente están por debajo del 10\% tanto en los esfuerzos como en la deformación.

TABLA V

COMPARACIÓN DE RESULTADOS EXPERIMENTALES VS TEÓRICOS

\begin{tabular}{ccccccccccc}
\hline \hline & \multicolumn{3}{c}{$\begin{array}{c}\text { VARIACIÓN } \\
\text { EXP - TEO }\end{array}$} & \multicolumn{3}{c}{$\begin{array}{c}\text { VARIACIÓN } \\
\text { SIM. CAE - TEO }\end{array}$} & \multicolumn{3}{c}{$\begin{array}{c}\text { VARIACIÓN } \\
\text { CAE }\end{array}$} \\
\hline $\mathrm{N}^{\circ}$ & $\delta$ & $\mathrm{s}_{\mathrm{n}}$ & $\mathrm{t}$ & $\delta$ & $\mathrm{s}_{\mathrm{n}}$ & $\mathrm{t}$ & $\delta$ & $\mathrm{s}_{\mathrm{n}}$ & $\mathrm{t}$ \\
$\mathrm{DE}$ & $(\%)$ & $(\%)$ & $(\%)$ & $(\%)$ & $(\%)$ & $(\%)$ & $(\%)$ & $\%$ & $\%$ \\
VIGA & & & & & & & & & \\
1 & 15,2 & 3,5 & 3,8 & 12,4 & 6,5 & 9,9 & 2,5 & 3,2 & 5,8 \\
2 & 13,8 & 0,98 & 1,3 & 11,7 & 6,0 & 6,5 & 1,8 & 5,0 & 5,1 \\
3 & 7,2 & 1,1 & 1,5 & 11,4 & 5,9 & 8,0 & 3,8 & 4,8 & 6,4 \\
\hline \hline
\end{tabular}

Comparando los valores experimentales con los teóricos, los valores de esfuerzos están bajo el 10\%, sin embargo, la deformación sobrepasa este límite. Se puede utilizar un factor de corrección para reducir esta variación. Este factor de corrección (fc) será el promedio de las razones entre los valores experimentales y teóricos de las tres vigas obteniendo como resultado fc=1.12.

\section{B. Análisis Económico}

En la Tabla VI se presentan los costos de fabricación de vigas completamente soldadas y las vigas ensayadas que tienen un porcentaje de soldadura de 37\%, 57\% y $70 \%$.

En el costo de fabricación solo se consideró como un valor de variación los costos de mano de obra y consumibles de soldadura (electrodo y gas de protección), los otros costos se consideran independientes del porcentaje de soldadura.

TABLA VI

COSTOS DE FABRICACIÓN DE LAS VIGAS

\begin{tabular}{|c|c|c|c|c|}
\hline Porcentaje de soldadura & $100 \%$ & $70 \%$ & $57 \%$ & $37 \%$ \\
\hline Mano de Obra (USD) & 25,51 & 24,96 & 26,54 & 28,40 \\
\hline Electrodo ER70S-6 (USD) & 8,33 & 5,83 & 4,71 & 3,08 \\
\hline $\begin{array}{l}\text { Gas de protección Ar+CO2 } \\
\text { (USD) }\end{array}$ & 5,18 & 3,62 & 2,92 & 1,92 \\
\hline COSTO TOTAL (USD) & 39,02 & 34,41 & 34,17 & 33,40 \\
\hline
\end{tabular}

Los costos totales de la Tabla anterior son para vigas de 3 metros de longitud, para realizar un mejor análisis se presentan los costos por metro de viga en la Tabla VII. 
TABLA VII

COSTOS DE FABRICACIÓN DE LAS VIGAS POR METRO

\begin{tabular}{cc}
\hline \hline Número de Viga & Costo total por metro (USD/m) \\
\hline Viga soldada (100\%) & 13,01 \\
Viga 3 (70\%) & 11,47 \\
Viga 2 (57\%) & 11,39 \\
Viga 1 (37\%) & 11,13 \\
\hline \hline
\end{tabular}

Si se realiza soldadura intermitente se obtiene una variación de entre el $13 \%$ y el $15 \%$ en los costos de fabricación respecto a una viga completamente soldada.

\section{CONCLUSIONES}

Al comparar los valores del esfuerzo normal, esfuerzo cortante y deformación entre los modelos experimental y teórico existe una variación del 3,51\%, 3,86\% y 15,25\% respectivamente, se concluye que el modelo teórico predice acertadamente los valores de los esfuerzos, sin embargo, sería de aplicar un factor de corrección para la deformación. El valor se calculó en 1,12 que corrige la variación disminuyéndola al 4,35\%. Dado que el esfuerzo normal, el esfuerzo cortante y la deformación tienen una relación inversamente proporcional al porcentaje de soldadura en la unión alma-patín mismas que presentan variaciones del $2.28 \%$ en el esfuerzo normal, 9,06\% en el esfuerzo cortante y $1,59 \%$ en la deformación, se establece que la variación en la longitud de los cordones de soldadura que unen alma y patín en una viga secundaria tipo I, no provoca una afectación significativa a la capacidad de carga y rigidez de la viga al ser sometida a cargas estáticas. Las vigas fabricadas con cordón de soldadura intermitente en comparación con las de cordón continuo presentaron una disminución en costo de consumibles para soldadura y mano de obra de $11,83 \%, 12,45 \%, 14,44 \%$ por cada metro de soldadura en vigas fabricadas con 37\%, 57\% y 70\% de soldadura respectivamente, otros rubros como los costos de material base, montaje, transporte, etc., no fueron tomados en cuenta ya que son iguales para vigas con soldadura continua o intermitente. En vigas secundarias sometidas a carga estática se podría reemplazar una soldadura continua por una intermitente en la unión de sus elementos, ya que, según el estudio realizado, esto no afectara al funcionamiento estructural de la viga.

\section{REFERENCIAS}

[1] Abarca, L. (Enero de 2016). TEC| Tecnológico de Costa Rica. Recuperado el 25 de marzo de 2017, de TEC| Tecnológico de Costa Rica: https://www.tec.ac.cr/en/proyectos/optimizacion-recursosmateriales-mano-obra-edi-caciones

[2] ANSI/AISC. (2010). Especificaciones ANSI/AISC 360-10 para Construcciones de Acero. Santiago de Chile: Asociación latinoamericana del Acero.

[3] ANSI/AWS. (2002). AWS D1.1 CÓDIGO DE SOLDADURA ESTRUCTURAL-ACERO (Español).

[4] Bresler, B., Lin, T., \& Scalzi, J. (1990). Diseño de Estructuras de Acero. México: LIMUSA.

[5] Budynas, R., \& Keith, N. (2008). Diseño en Ingeniería Mecánica de Shigley. México: Mc Graw Hill.

[6] McCormac, J. C. (2013). Diseño de Estructuras Metálicas. México DF: Alfaomega.

[7] NEC-SE-AC. (2014). Código Ecuatoriano de la Construcción Estructuras de acero. Quito: Dirección de Comunicación Social, MIDUVI.

[8] Reza, M., Rastani, M., \& Ghavami, K. (2006). Numerical study on the permissible gap of intermittent fillet welds of longitudinally stiffened plates under in plane axial compression. Journal of Constructional Steel Research, 1415-1428.

[9] Toyoda, M., \& Mochizuki, M. (2004). Control of mechanical properties in structural steel welds by numerical simulation of coupling among temperature, microstructure, and macro-mechanics. Science and Technology of Advanced Materials, 255-266. 